\title{
SIMS2018 CFD Simulation of Solidification of non-Newtonian Fluid Flowing in a Complex Geometry Pipeline in Turbulent Flow Regime
}

\author{
Ludmila Vesjolaja $^{1} \quad$ Jakub M. Bujalski $^{2} \quad$ Knut Vaagsaether $^{1}$ \\ ${ }^{1}$ Department of Process, Energy and Environmental Technology, University of South-Eastern Norway, Norway, \\ \{ludmila.vesjolaja, knut.vagsather\}@usn.no \\ ${ }^{2}$ Process Modeling and Control Department, Yara Technology Centre, Norway, jakub.bujalski@yara.com
}

\begin{abstract}
In this CFD (Computational Fluid Dynamics) study, the turbulent flow of a non-Newtonian fluid through an industrial scale transportation pipeline is modelled in Ansys Fluent ${ }^{\circledR}$, with a focus on the fluid solidification due to heat transfer on the pipe walls. The turbulence was modelled using two different turbulence models: a standard low-Reynolds-number $k-\varepsilon$ turbulence ChanHsieh-Chen (CHC) model and a modified Malin's turbulence model. Simulations were performed with fluid viscosity depending both on the shear rate as well as on the temperature. However, according to the simulation results, as long as the inlet fluid velocity is maintained sufficiently high (turbulent flow), the occurrence of fluid solidification is not significantly affected by the viscosity dependence on the temperature. All turbulence models show fluid solidification on the pipe walls, and not inside the pipe itself. The standard CHC model shows more pipe wall zones that are solidified, while the modified Malin's turbulence model shows a more diffusive behavior. The latter model has an effect on the velocity distribution across the pipeline in such a way that the fluid flow between the pipelines become more evenly distributed. The simulation results of pipe insulation and liquid flow rate, on the fluid solidification were used to give recommendations of improvements to avoid blockages in the transportation pipelines in the industrial process. According to the simulation results, the use of pipe insulation can minimize the occurrence of fluid solidification on the pipe walls.
\end{abstract}

Keywords: non-Newtonian fluid, turbulent pipe flow, solidification, Malin turbulence model, CFD

\section{Introduction}

The solidification of fluids in pipe flows is an important topic in many practical engineering problems, especially in manufacturing industries where material phasechange may occur. The change of phase of the fluid may cause damages to the pipelines due to blockages that may eventually lead to unforeseen plant shut downs and additional cleaning procedures. Due to heat transfer the phase-change of the fluid usually occurs first on the pipe wall where a solid phase develops and increases its radial size with time causing possible pipeline blockages (Conda et al., 2004; Wei and Güceri, 1988). The modelling of this phenomenon is very challenging since they are time-dependent and factors such as flowrate and temperature directly affect its formation.

Despite the problems caused by fluid solidification in various engineering processes, very few research works about fluid solidification have been published in literature. Early studies devoted to the solidification phenomena were performed by Hirschberg (1962) and by Zerkle and Sunderland (1969). In these works, the solidification of the fluid was studied by assuming a laminar flow regime at the steady-state (Hirschberg, 1962; Zerkle and Sunderland, 1969). Wei and Güceri (1988) conducted another significant study where an attempt was made to develop a numerical model for describing the solidification in fully developed internal pipe flows. Almost 20 years later, Conde et al. (2004) have developed a 2D numerical model for describing the solidification of water, olive oil and aluminum in cylindrical ducts. Interestingly, this is the only available study where solidification in internal pipe flows was conducted using Ansys Fluent ${ }^{\circledR}$. During the past ten years only few works were devoted to the solidification in internal flows, and these mostly focus on the enhancement of phase-change in heat pipes (Motahar and Khodabandeh, 2016; Sharifi et al., 2014). To our knowledge, only Myers and Low (2013) has published on the solidification of the non-Newtonian fluid flows in pipes. They have developed a mathematical model for the solidification of the Power-Law fluids (shearthinning) in narrow pipes and have assumed a laminar flow regime as well as using MATLAB to solve their model equations (Myers and Low 2013).

This study investigates the solidification during fluid transport around a final stage of fertilizer particulation process. One of the challenges faced in the manufacturing of complex fertilizers is the efficient transportation of the process fluids from one stage of the process operation to the next. This relatively simple 
operation can be a major issue as the fluid has nonNewtonian behavior. The complex behavior of the process fluid affects the efficiency of pumps causing pipe blockages due to pre solidification in the pipes, leading to loss of production. The understanding of this rheological behavior of the non-Newtonian fluid is of fundamental importance for proper operation of the plant. Detailed knowledge of non-Newtonian fluid solidification can also be useful for designing layout of the pipelines and for proper selection of pipe insulation to reduce the risk of pipe blockages. Hence, in this paper, the authors have contributed with the modeling and numerical simulations of the solidification of a highly non-Newtonian fluid in turbulent flow regime in 3D. The focus is on the flow of an industrial case nonNewtonian fluid through complex geometry pipelines in turbulent flow regime.

\section{Model development}

\subsection{Solidification of the fluid}

In this study, "Enthalpy-Porosity" formulation is used to model fluid solidification. This approach is based on the studies by Voller and Prakash (1987) and the method is also available in Ansys Fluent ${ }^{\circledR}$ User`s guide (2006). According to this technique, the melt interface is computed implicitly. In this method, a liquid fraction that is linked with every cell in the computational domain is used to track the interface. The liquid fraction indicates the fraction of the cell that is in the liquid state. Ansys Fluent ${ }^{\circledR}$ uses the "mushy zone" which is modelled as a "pseudo-porous media" in which the porosity (or liquid fraction) ranges from one to zero. When the porosity is equal to one, the fluid is in fully liquid-state, and when it is equal to zero, the fluid is in solid-like state with zero velocity (Ansys Fluent ${ }^{\mathbb{R}}$, 2006).

The corresponding energy equation and enthalpy formulations solved in Ansys Fluent ${ }^{\circledR}$ (2006) are represented with Equation 1 and Equations 2 to 4 respectively. The liquid fraction is calculated using Equation 5 and the solution for the temperature is found iteratively using Equations 1 to 5:

$$
\begin{aligned}
& \frac{\partial}{\partial t}(\rho H)+\operatorname{div}(\bar{v} \rho H)=\operatorname{div}(k \operatorname{grad} T)+S_{t} \\
& H=h+\Delta H \\
& h=h_{r e f}+\int_{T_{r e f}}^{T} c_{p} d T \\
& \Delta H=\beta L
\end{aligned}
$$$$
\beta=\left\{\begin{array}{c}
0, \text { if } T<T_{\text {solid }} \\
1, \text { if } T>T_{\text {liquid }} \\
\frac{\left(T-T_{\text {solid }}\right)}{\left(T_{\text {liquid }}-T_{\text {solid }}\right)}
\end{array}\right.
$$

where $\rho$ is density, $H$ is total enthalpy, $\bar{v}$ is velocity vector, $k$ is turbulent kinetic energy, $T$ and is absolute temperature, $S_{t}$ is shear rate component, $h$ is sensible enthalpy, $\Delta H$ is latent heat, and $c_{p}$ is specific heat.

\subsection{Rheology of the fluid}

The particular fluid used in this study is a shear-thinning slurry whose viscosity depends on both the shear rate and the temperature. The rheological properties of the fluid taken into consideration were obtained experimentally using Anton Paar Modular Compact Rheometer 302. The experimentally obtained flow curve was fitted to different rheological models. The

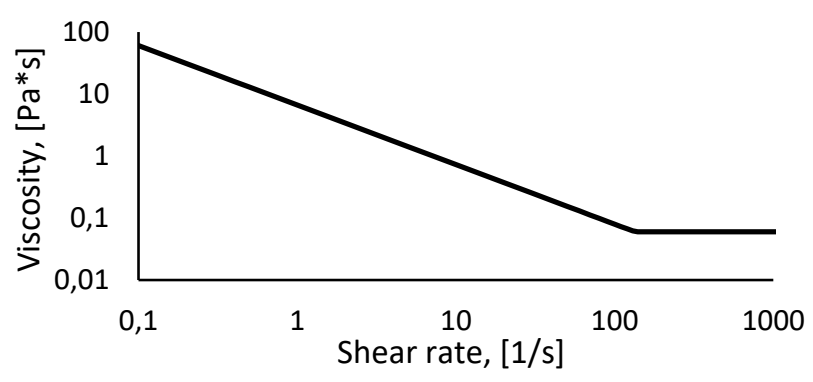

Figure 1. Fluid viscosity dependence on the applied shear rate (log-log scale).

best fit was obtained with the Power-Law model for non-Newtonian fluids. The fitted flow curve used in this study is illustrated in Figure 1.

The effect of the temperature on the viscosity of the fluid was characterized using cooling curve illustrated in Figure 2. It indicates that the temperature significantly affects the viscosity of the given fluid. The higher the temperature, the lower is the apparent viscosity of the fluid. Therefore, to obtain a better model of the given process, the changes in the viscosity due to the temperature, in combination with its dependence on the shear rate, should be included when modelling solidification.

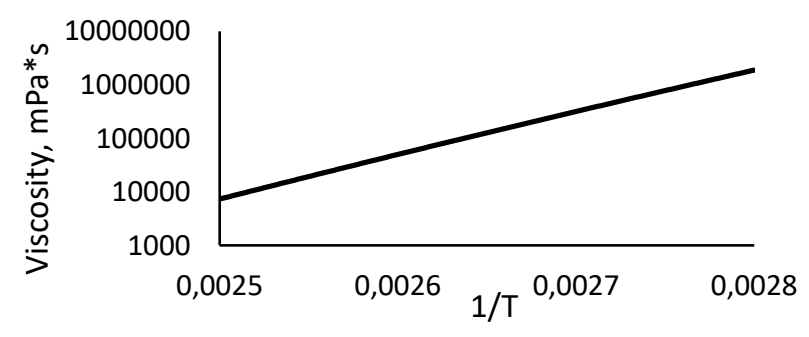

Figure 2. Fluid viscosity dependence on temperature.

It is possible to model the effect of both the temperature and the shear rate on the viscosity of the fluids in Ansys Fluent ${ }^{\circledR}$. For this, an activation energy constant (also known as activation temperature) and a reference temperature should be known. In this study, the activation temperature was estimated using the Least Square fitting method. Least Square fitting was performed by using the modified Arrhenius law (Equation 6) showed in Rojas et al. (2008) and was fitted to the cooling curve polynomial (Figure 2) obtained 
experimentally. The estimated value of the activation temperature $(\alpha)$ is listed in Table 2.

$\mu=\mu_{\infty} \exp \left(\frac{E}{R T}\right)$

where $\mu$ is apparent viscosity, $\mu_{\infty}$ is infinite viscosity, $E$ is energy, and $R$ is universal gas constant.

\subsection{Turbulence model}

The standard $k-\varepsilon$ turbulence model is widely used in modelling of internal turbulent pipe flows due to its simplicity and applicability. However, the standard $k-\varepsilon$ turbulence model does not account for drag reduction effect and may have unsatisfactory results and predictions at the near wall zones where eddy viscosity changes rapidly with the distance from the pipe wall (Mathur and He, 2013; Versteeg and Malalasekera, 2007). This might have a crucial role in modelling solidification since the solidification of the fluid is expected to occur on pipe walls. One solution to overcome this problem is to use low-Reynolds-number $\mathrm{k}-\varepsilon$ turbulence models that are specifically developed to account for near-wall phenomena. However, the fluid taken into consideration exhibits a highly nonNewtonian behaviour. To account for this phenomena, in this paper Malin's turbulence model for Power-Law fluids has been modified (Vesjolaja, 2016).

Malin (Malin, 1997; Malin, 1998) has developed a model where the damping functions are specially treated to describe the non-Newtonian fluid flow in the turbulent flow regime (Reynolds number (Re) up to $10^{5}$ ). Malin's model is based on low-Reynolds-number $k-\varepsilon$ turbulence models like the Lam-Bremhost (LB) model (Lam and Bremhost, 1981). The only and the most important difference between these two models is in the way the eddy/turbulent viscosity is calculated. For both models, the transport equation for the kinetic energy is formulated as in the standard $k-\varepsilon$ turbulence models and can be founded in earlier works (Malin, 1997; Lam and Bremhost, 1981). However, the eddy dissipation rate formulation that carries turbulent viscosity term differs between the two models. With the Malin's turbulence model, the formulation of eddy viscosity includes the power-law index (that carries the
non-Newtonian characteristics) in the damping function term, while this is not present in the Lam-Bremhost model.

The transport equation for the eddy dissipation rate is given by Equation 7, meanwhile the turbulent viscosity is calculated by Equation 8 using damping functions $\left(f_{1}, f_{2}\right.$ and $\left.f_{\mu}\right)$ defined in Table 1 (Malin, 1997).

$$
\begin{aligned}
& \frac{\partial(\rho \varepsilon)}{\partial t}+\operatorname{div}(\rho \varepsilon U)=\operatorname{div}\left(\frac{\mu_{t}}{\sigma_{\varepsilon}} \operatorname{grad} \varepsilon\right)+ \\
& f_{1} C_{1} \frac{\varepsilon}{k} 2 \mu_{t} S . S-f_{2} C_{2} \rho \frac{\varepsilon^{2}}{k} \\
& \mu_{t}=C_{\mu} f_{\mu} k^{2} / \varepsilon
\end{aligned}
$$

where $\varepsilon$ is turbulent dissipation rate, $U$ is velocity component, $\mu_{t}$ is turbulent viscosity, and $C_{1}, C_{2}$ and $C_{\mu}$ are turbulence adjustable constants.

Malin's turbulence model is not readily available in Ansys Fluent ${ }^{\circledR}$. It is possible to implement Malin's turbulent model by using User Defined Functions (UDF), however the computational time for the convergence of the solution is prolonged significantly. The increased computational time is even more pronounced when modelling thermal effects (solidification in this case). Hence, the Malin's turbulence model was implemented in a robust way by enabling and modifying the built-in low-Reynoldsnumber k- $\varepsilon$ model. For this, Malin's eddy viscosity formulation was coupled to the built-in CHC (Chang et al., 1995) turbulence model using a UDF. The CHC model was preferred to the LB model as well to other turbulence models available in Ansys Fluent ${ }^{\circledR}$ e.g. Launder-Sharma, Abid, Yang-Shih and the AbeKondoh-Nagano models. This choice is based on the fact, also supported by previous studies (Vesjolaja, 2016) that the CHC model exhibits more stable solutions during the simulations. Both the standard $\mathrm{CHC}$ and the modified Malin's models were compared in this study (see Equations 7, 8 and Table 1 for the algebraic

\begin{tabular}{|c|c|c|c|c|}
\hline Turbulence Model & $\overline{f_{1}}$ & $\overline{f_{2}}$ & $\overline{f_{\mu}}$ & $\begin{array}{c}\text { Wall } \\
\text { boundary } \\
\text { conditions }\end{array}$ \\
\hline $\begin{array}{l}\text { Malin original } \\
\text { (Malin, 1997) }\end{array}$ & $1+\left(\frac{0.05}{f_{\mu}}\right)^{3}$ & $1-\exp \left(-\mathrm{Re}_{\mathrm{t}}^{2}\right)$ & $\begin{array}{c}\left(1-\exp \left(-0.0165 \mathrm{Re}_{\mathrm{k}} / \mathrm{n}^{1 / 4}\right)\right)^{2} * \\
\left(1+20.5 / \mathrm{Re}_{\mathrm{t}}\right)\end{array}$ & $\frac{\partial \varepsilon}{\partial y}=0$ \\
\hline $\begin{array}{l}\text { CHC original } \\
\text { (Chang et al., } \\
\text { 1995) }\end{array}$ & 1.0 & $\begin{array}{l}\left(1-0.01 \exp \left(-\mathrm{Re}_{\mathrm{t}}^{2}\right)\right) \\
*\left(1-\exp \left(-0.0631 \mathrm{Re}_{\mathrm{k}}\right)\right)\end{array}$ & $\begin{array}{c}\left(1-\exp \left(-0.0215 \operatorname{Re}_{\mathrm{k}}\right)\right)^{2} \\
\left.31.66 / R e_{t}^{5 / 4}\right)\end{array}$ & $\varepsilon_{w}=v\left(\frac{\partial^{2} k}{\partial y^{2}}\right)$ \\
\hline $\begin{array}{l}\text { Modified Malin’s } \\
\text { (Vesjolaja, 2016) }\end{array}$ & 1.0 & $\begin{array}{l}\left(1-0.01 \exp \left(-\mathrm{Re}_{\mathrm{t}}^{2}\right)\right) *(1 \\
\left.-\exp \left(-0.0631 \mathrm{R}_{\mathrm{k}}\right)\right)\end{array}$ & $\begin{array}{c}\left(1-\exp \left(-0.0165 \mathrm{Re}_{\mathrm{k}} / \mathrm{n}^{1 / 4}\right)\right)^{2} * \\
\left(1+20.5 / \mathrm{Re}_{\mathrm{t}}\right)\end{array}$ & $\varepsilon_{w}=v\left(\frac{\partial^{2} k}{\partial y^{2}}\right)$ \\
\hline
\end{tabular}
equations used in different models).

Table 1. Turbulence model damping functions used in Equations 7 and 8. 


\section{Fertilizer process as a case study}

A case study has been performed where the modified Malin's model and the CHC model described in Section 2.2 is used to simulate the flow of a non-Newtonian fluid in 3D. The case study consists of the flow through a bended pipeline as shown in Figure 3. Geometry of the pipelines for the process taken into consideration.Figure 3 and is a part of a process from a fertilizer production plant.

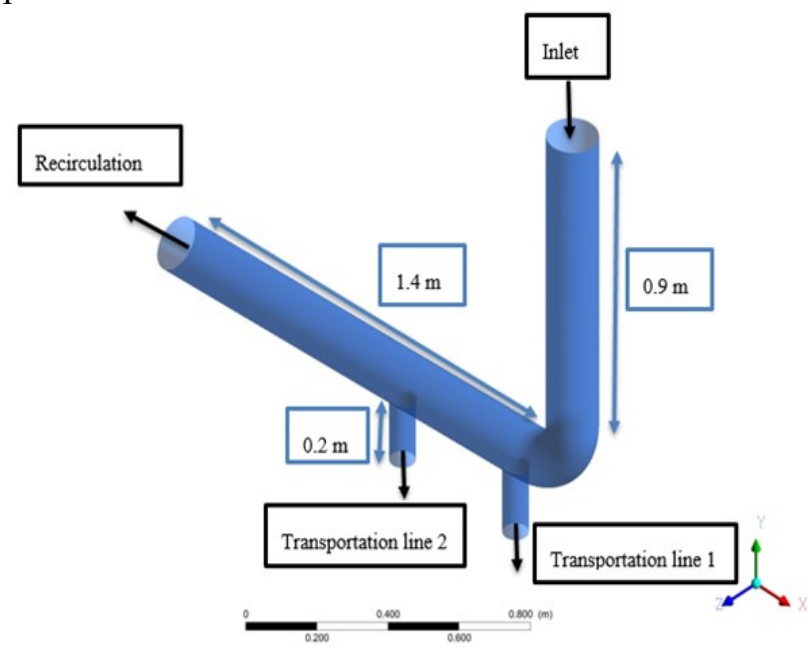

Figure 3. Geometry of the pipelines for the process taken into consideration.

The pipework consists of the main pipeline which has a bend of $90^{\circ}$ and two additional pipelines (denoted as "Transportation line 1" and "Transportation line 2" in Figure 3) that are attached right after the main pipeline bend. The diameter of the main pipeline is $150 \mathrm{~mm}$ and that of the two transportation lines is $75 \mathrm{~mm}$ each. The thickness of the pipe walls is $5 \mathrm{~mm}$ and the pipe material is stainless steel. The characteristics of the pipe wall material are listed in Table 2.

The process fluid is pumped through the main pipeline inlet (denoted as "Inlet" in Figure 3) and is transported to the next stage of operation through the Transportation lines 1 and 2. The remaining process fluid is recirculated using the "Recirculation" line as denoted in Figure 3.

During the operation of the fertilizer plant, reduced pump capacity at the inlet of the pipe was observed. It is suspected that this could be due to the solidification of the fluid in the pipelines. This study thus focuses on understanding as to where and how such solidification occurs in the pipelines. For this, a suitable model capable of taking into account the rheology of the nonNewtonian fluid is simulated in Ansys Fluent ${ }^{\circledR}$. Detailed 3D simulations have been performed to observe where and how such solidification occurs for a given operating condition of the process giving a result for steady state under the selected boundary conditions.

\section{Methods and materials}

General settings: The simulation of the given process were performed using commercial CFD tool, Ansys Fluent $^{\mathbb{R}}$ Academic Research, Release 16.2. The geometry was designed using Ansys DesignModeler and mesh was generated using sweep mesh method with the element size of $5 \mathrm{~mm}$ and $10^{\circ}$ curvatures (1681179 cells in the simulation domain). The simulations were performed for the 3D steady-state regime using pressure based solver and absolute velocity formulation.

Momentum Boundary Conditions: The inlet velocities were varied from $3.0 \mathrm{~m} / \mathrm{s}$ to $5.0 \mathrm{~m} / \mathrm{s}$ in accordance to the different simulation case studies. The inlet velocities were defined to be the same over the whole cross sectional area of the pipe inlet. The pipeline outflows were defined as pressure outlets and gauge pressure was set to zero.

The estimation of the Reynolds-number for the internal pipe flow under consideration is challenging due to the non-Newtonian behavior of the fluid. In this study, the "generalized Reynolds-number" has been calculated using the Dodge and Metzner correlation (Dodge and Metzner, 1959) for the Power-Law fluids (Equation 9). For the calculation of the Reynoldsnumber, the inlet velocity $\left(v_{\text {bulk }}\right)$ was taken as a bulk velocity.

$$
R e=\frac{\left(\rho * v_{b u l k}^{2-n} * D^{n}\right)}{K\left(0.75+\frac{0.25}{n}\right)^{n} * 8^{n-1}}
$$

where $D$ is diameter of the pipe, $n$ is non-Newtonian index, $K$ is consistency index.

Turbulence model: Turbulent flow was modelled using the standard $\mathrm{CHC}$ turbulence model and the modified Malin's model as described in Section 2.3. The modified Malin's turbulence model was implemented using UDF.

Thermal Boundary Conditions: The inlet fluid temperature as well as the temperature for the backflow

Table 2. Thermo-physical properties of the materials used in this study.

\begin{tabular}{|c|ccccccc|}
\hline Material & $\begin{array}{c}\rho \\
\left(\mathrm{kg} / \mathrm{m}^{3}\right)\end{array}$ & $\begin{array}{c}C_{p} \\
(\mathrm{~J} /(\mathrm{kg} \cdot \mathrm{K}))\end{array}$ & $\begin{array}{c}\mathrm{k}_{\text {th }} \\
(\mathrm{W} /(\mathrm{m} \cdot \mathrm{K}))\end{array}$ & $\begin{array}{c}T_{\text {liq }} \\
(\mathrm{K})\end{array}$ & $\begin{array}{c}T_{\text {sol }} \\
(\mathrm{K})\end{array}$ & $\begin{array}{c}T_{\text {ref }} \\
(\mathrm{K})\end{array}$ & $\begin{array}{c}\alpha \\
(\mathrm{K})\end{array}$ \\
\hline $\begin{array}{c}\text { Fluid } \\
\text { Pipe Wall }\end{array}$ & 1400 & 1470 & 0.3 & 403 & 393 & 303 & 18100 \\
$\begin{array}{c}\text { (stainless steel) } \\
\begin{array}{c}\text { Wall insulation } \\
\text { (calcium silicate) }\end{array}\end{array}$ & 7900 & 515 & 16.6 & Not used & Not used & Not used & Not used \\
\hline
\end{tabular}


of the fluid at the outlets was set to $413 \mathrm{~K}$. The wall heat transfer was modelled using "Thin Wall approach" and the wall temperature was set to $296 \mathrm{~K}$.

Solution methods: The simulations were performed in a steady-state regime. The Semi-Implicit Method for Pressure Linked Equations (SIMPLE) method was used as a pressure-velocity coupling scheme. The first order upwind scheme was chosen as a spatial discretization method. The standard solution initialization was used for the standard CHC turbulence model. However, when simulating with the modified Malin's turbulence model, the solution was initialized with the end results of the corresponding $\mathrm{CHC}$ model (simulated with the standard settings). The mesh sensitivity study was performed and the solution was assumed to be converged when the scaled residuals become constant. In addition, mass flowrates were checked and the solution was assumed to be converged if the mass flowrate imbalance was less than $10^{-4}$. Mass outflow rates were computed using Surface Integrals available in Ansys Fluent ${ }^{\circledR}$.

Material properties: The non-Newtonian behavior of the fluid was characterized with the Power-Law model for non-Newtonian fluids $(n=0.05)$. The thermophysical properties of the fluid and the wall material (including wall insulation) used for simulations are summarized in Table 2.

\section{Results and Discussion}

\subsection{Effect of temperature on fluid's viscosity}

To have a better understanding of how the temperature affects the viscosity of the non-Newtonian fluid and how this further influences the fluid solidification, simulations were modelled using standard CHC turbulence model. To start with, the fluid flow rate corresponding to the transient flow regime (with $\mathrm{Re}=$ 6796 and the inlet velocity of $3.0 \mathrm{~m} / \mathrm{s}$ ) was considered. Two cases were studied: (a) viscosity dependent only on the shear rate and, (b) viscosity dependent on both the temperature and the shear rate, and results are shown in Figure 4.

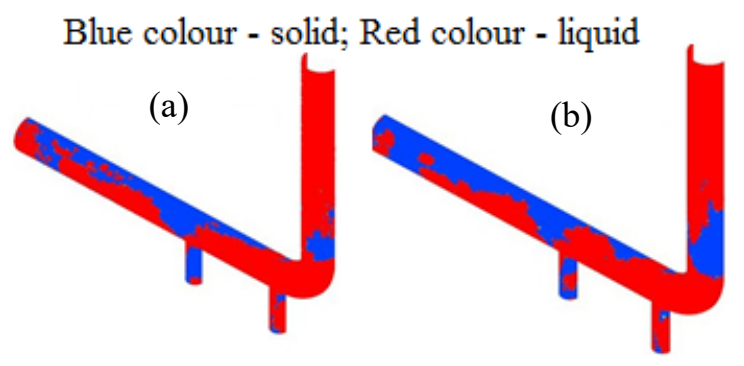

Figure 4. Solidification profiles using $\mathrm{CHC}$ model with 3 $\mathrm{m} / \mathrm{s}$ inlet velocity for fluid with: (a) shear rate dependent viscosity; (b) both shear rate and temperature dependent viscosity.

Both simulations showed fluid solidification on the pipe walls, meanwhile no solidification occurred inside the pipe itself for both cases. Moreover, at the pipe walls, observable differences in the solidification profiles were seen for the two cases. For the first case (viscosity not affected by temperature), smaller solidification zones were observed (Figure 4a) compared to the results obtained for the latter case (see Figure 4b).

When the flow was fully turbulent in nature $(\mathrm{Re}=$ 18497 with inlet velocity $5 \mathrm{~m} / \mathrm{s}$ ), the solidification profiles between the two cases did not reveal any significant differences (Figure 5). This might be due to the generation of higher turbulence inside the pipe and hence higher eddy viscosity. Therefore, changes in the molecular viscosity will not affect the solidification results significantly. Hence, if there are no perturbations in the actual process inlet velocity and is maintained at $5 \mathrm{~m} / \mathrm{s}$, the fluid solidification will not be significantly affected by the viscosity dependency on the temperature. Furthermore, for performing the simulations, the computational time is significantly smaller for case (a) than for case (b). Due to these reasons, the only case (a) will be studied further on and case (b) will not be discussed in the remaining part of this paper.

\section{Blue colour - solid; Red colour - liquid}

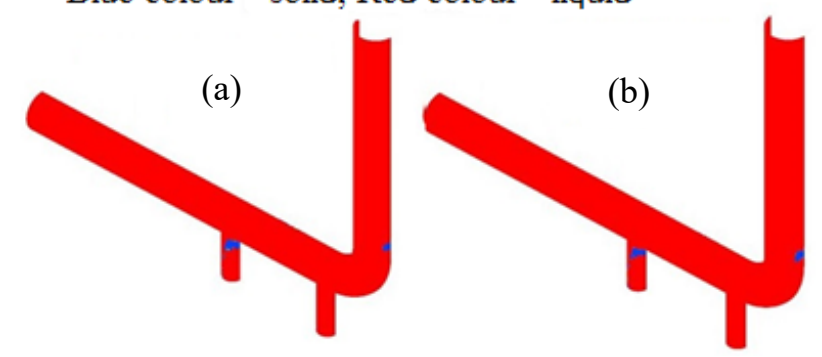

Figure 5. Solidification profiles using $\mathrm{CHC}$ model with 5 $\mathrm{m} / \mathrm{s}$ inlet velocity for fluid with: (a) shear rate dependent viscosity; (b) both shear rate and temperature dependent viscosity.

\subsection{Different turbulence models for solidification}

The solidification of the fluid was studied using the standard CHC and the modified Malin's turbulence models. The viscosity dependency on the temperature was ignored in the simulations due to the reasons described in Section 5.1.

Both the standard CHC and the modified Malin's turbulence models showed fluid solidification on the pipe walls, meanwhile no solidification occurred inside the pipe itself. However, the location of the solidification zones and their sizes were different for these two models.

The standard CHC model showed more pipe wall zones that are susceptible to fluid solidification. The "risk zones", i.e. the zones where the probability of the occurrence of fluid solidification is higher are the pipe wall towards the main pipe outlet, the transportation 
pipeline walls and the backside of the pipe wall just before the main pipe bend (denoted as "zone 1", "zone 2 " and "zone 3" respectively in Figure 6). The occurrence of the fluid solidification on "zone 1" can be explained with the presence of a larger surface area of the wall that is exposed to the ambient air temperature. The larger the surface area, the larger will be the heat transfer between the ambient air and the fluid and hence more fluid will be solidified. Solidifications on "zone 2" and "zone 3" can be explained with the occurrence of lower fluid velocities at these zones. The lower the velocity, the slower the fluid molecules are moving inside the pipe and hence these molecules have comparatively more time to exchange heat with the ambient air through the pipe walls.

The modified Malin's turbulence model showed different and smaller "risk zones" for solidification compared to the CHC model. The solidification of the fluid occurred after the main pipe bend and near the outlet of the transportation Pipelines 1 and 2 (denoted as "zone 4" and "zone 5" respectively in Figure 7). From the velocity profiles it can be seen that these zones are the low-velocity zones or the "dead zones".

The occurrence of solidification on these zones can also be explained with a longer residence time of the fluid molecules (due to lower velocity) in these zones and hence more transfer of heat from the fluid to the ambient air through the pipe walls. Interestingly in contrast to the standard CHC model, the modified
Malin's model can capture these "dead zones" which are the "risk zones" for fluid solidification. The occurrence of less solidification along the pipeline walls can be explained with the diffusive behaviour of the modified Malin's model (see velocity profiles on Figure 6a and Figure 7a). The higher the turbulence, the higher is the velocity and hence the less is the residence time of the fluid molecule inside the pipeline. Therefore, this causes fewer fluid molecules to solidify on the pipe walls. The modified Malin's model shows more evenly distributed mass outflow rates and hence evenly distributed velocities along the pipelines.

\subsection{Effect of pipe wall insulation on solidification}

To investigate if the insulation of the pipelines would minimize the occurrence of fluid solidification, the simulations were also performed with the changes in the thickness of the pipe wall insulation. The properties of the insulation layer are summarized in Table 2. The solidification was simulated using both the standard CHC and the modified Malin's turbulence models.

Both turbulence models showed similar simulation results revealing that no solidification occurred when the pipe wall was insulated with a $50 \mathrm{~mm}$-thick insulation layer jacket. This behavior was observed for the higher fluid velocity $(5 \mathrm{~m} / \mathrm{s})$ as well as for the lower fluid velocity $(3 \mathrm{~m} / \mathrm{s})$. However, without pipe insulation, the fluid solidifies at different places along the pipeline
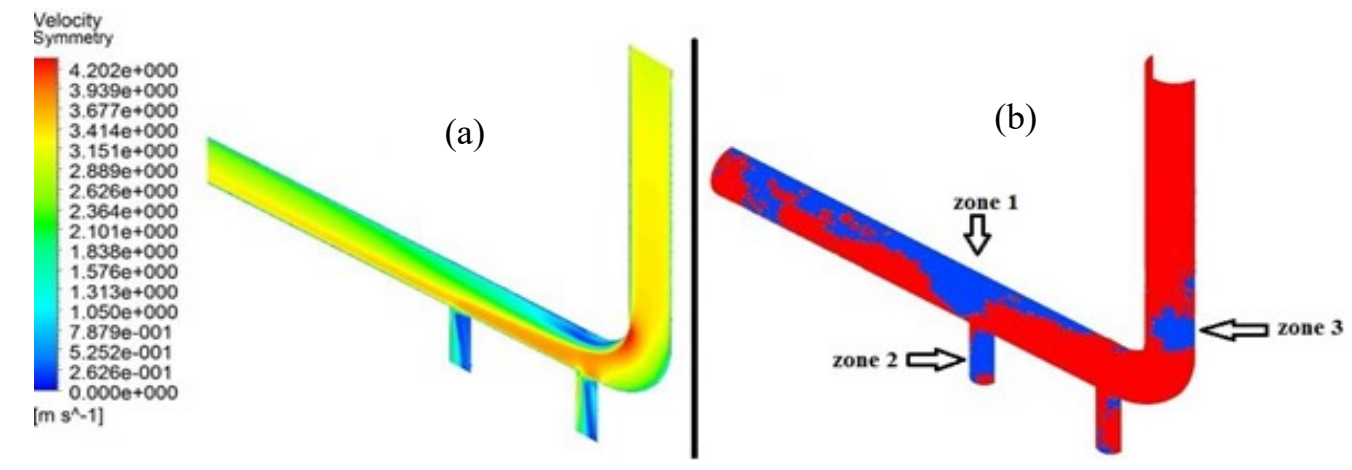

Figure 6. Simulation results with the $\mathrm{CHC}$ model ( $3 \mathrm{~m} / \mathrm{s}$ inlet velocity): (a) velocity profile; (b) fluid solidification.

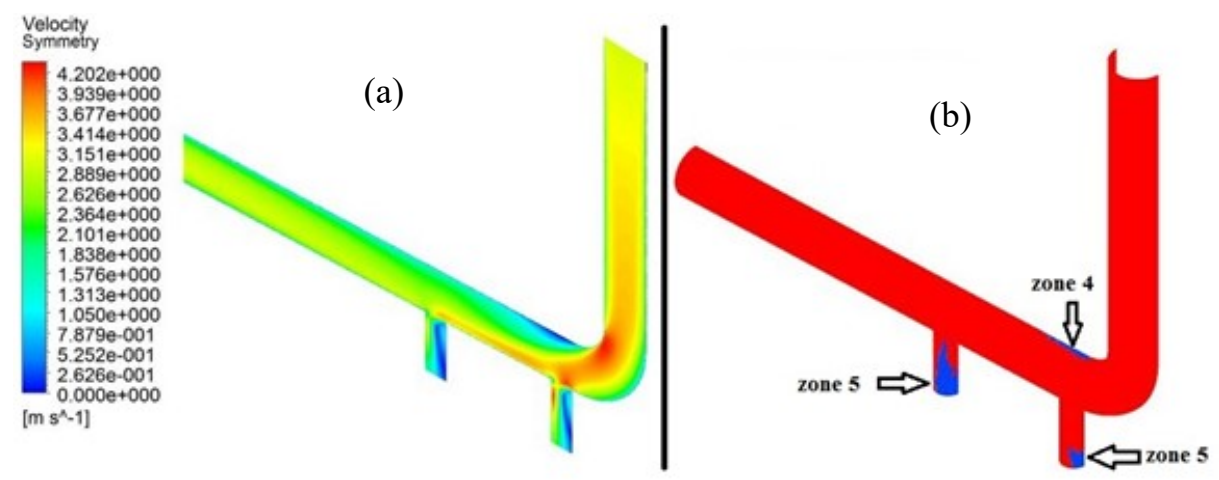

Figure 7. Simulation results with the modified Malin's model ( $3 \mathrm{~m} / \mathrm{s}$ inlet velocity): (a) velocity profile; (b) fluid solidification. 
as described in the previous sections. Thus, the absence of the pipe wall insulation could be a probable reason for the detected reduced pump capacity during the operation process.

\section{Conclusions}

The solidification model was coupled to the nonNewtonian turbulence model in Ansys Fluent ${ }^{\circledR}$ by using UDFs. The "Enthalpy-Porosity" formulation was used to model fluid solidification, meanwhile modified Malin's model was used to model turbulent fluid flow. The Malin's eddy viscosity model was combined with the low-Reynolds-number $k-\varepsilon$ turbulence Chan-HsiehChen model to account for the non-Newtonian behaviour of the process fluid.

As long as the inlet fluid velocity is maintained sufficiently high (turbulent flow), the occurrence of fluid solidification is not significantly affected by the viscosity dependence on the temperature. According to the simulation results, both the $\mathrm{CHC}$ and modified Malin's turbulence models showed fluid solidification on the pipe walls, and not inside the pipe itself. The location and the size of fluid solidification zones were different for these two models. Modified Malin's model showed more diffusive behaviour. It is difficult to conclude which of these models better represents the real process. Validation of the simulation results should be considered as a potential future work.

According to the simulation results, the use of pipe insulation can minimize the occurrence of fluid solidification on the pipe walls. Without insulation, solidification occurred on the pipe walls. Thus, the use of pipe insulation is beneficial and is highly recommended for the investigated process.

\section{Acknowledgements}

Authors kindly thank the department of Process Modelling and Control at Yara International ASA for providing the knowledge about the process. We would also like to thank the NPK department at Yara specially Carole Allen for her contributions in performing experiments.

\section{References}

Chang, K., Hsieh, W., \& Chen, C. (1995). A modified lowReynolds-number turbulence model applicable to recirculating flow in pipe expansion. Journal of fluids engineering, 117(3), 417-423.

Conde, R., Parra, M., Castro, F., Villafruela, J., Rodríguez, M., \& Méndez, C. (2004). Numerical model for two-phase solidification problem in a pipe. Applied Thermal Engineering, 24(17), 2501-2509.

Dodge, D., \& Metzner, A. (1959). Turbulent flow of nonNewtonian systems. AIChE Journal, 5(2), 189-204.

Ansys Fluent ${ }^{\circledR} 6.3$ User's Guide, (2006).
Hirschberg, H. (1962). Freezing of piping systems. Kaltetechnik, 14, 314-321.

Lam, C., \& Bremhost, K. (1981). A modified form of the k- $\varepsilon$ model for predicting wall turbulence. ASME J. Fluids Eng, 103, 456-460.

Malin, M. (1997). Turbulent pipe flow of power-law fluids. International communications in heat and mass transfer, 24(7), 977-988.

Malin, M. (1998). Turbulent pipe flow of Herschel-Bulkley fluids. International communications in heat and mass transfer, 25(3), 321-330.

Mathur, A., \& He, S. (2013). Performance and implementation of the Launder-Sharma low-Reynolds number turbulence model. Computers \& Fluids, 79, 134139.

Motahar, S., \& Khodabandeh, R. (2016). Experimental study on the melting and solidification of a phase change material enhanced by heat pipe. International communications in heat and mass transfer, 73, 1-6.

Myers, T., \& Low, J. (2013). Modelling the solidification of a power-law fluid flowing through a narrow pipe. International Journal of Thermal Sciences, 70, 127-131.

Rojas, M. A., Castagna, J., Krishnamoorti, R., \& Han Dh, T. A. (2008). Shear thinning behavior of heavy oil samples: laboratory measurements and modeling. Paper presented at the SEG Annual Meeting, Las Vegas.

Sharifi, N., Bergman, T. L., Allen, M. J., \& Faghri, A. (2014). Melting and solidification enhancement using a combined heat pipe, foil approach. International Journal of Heat and Mass Transfer, 78, 930-941.

Versteeg, H. K., \& Malalasekera, W. (2007). An introduction to computational fluid dynamics: the finite volume method: Pearson Education.

Vesjolaja, L. (2016). Flow Patterns of Highly Non-Newtonian Fluids in Industrial Applications. Master Thesis in Process Technology, Department of Process, Energy and Environmental Technology, University College of Southeast Norway.

Voller, V. R., \& Prakash, C. (1987). A fixed grid numerical modelling methodology for convection-diffusion mushy region phase-change problems. International Journal of Heat and Mass Transfer, 30(8), 1709-1719.

Wei, S.-S., \& Güceri, S. I. (1988). Solidification in developing pipe flows. International Journal of Heat and Fluid Flow, 9(2), 225-232.

Zerkle, R. D., \& Sunderland, J. (1968). The effect of liquid solidification in a tube upon laminar-flow heat transfer and pressure drop. Journal of Heat Transfer, 90(2), 183-189. 